\title{
Clinical utility and patient considerations in the use of the sitagliptin-metformin combination in Chinese patients
}

This article was published in the following Dove Press journal:

Patient Preference and Adherence

9 February 2015

Number of times this article has been viewed

\author{
Qiang Du \\ Yan-Jun Wang \\ Sheng Yang \\ Ping Han \\ Department of Endocrinology, \\ Shengjing Hospital of China Medical \\ University, Shenyang, Liaoning, \\ People's Republic of China
}

Correspondence: Qiang Du Department of Endocrinology, Shengjing Hospital of China Medical University, Shenyang I I0004, Liaoning, People's Republic of China

Tel +86 I3842060676

Email kobe9903@sina.com

\begin{abstract}
The prevalence of diabetes mellitus (DM) continues to increase each year. However, the efficacy of glucose-lowering therapies remains unsatisfactory. Moreover, the clinical characteristics and manifestations of DM in Chinese patients are different from those in Western patients. Thus, it is imperative to develop an optimal treatment protocol for lowering blood glucose levels in Chinese patients with DM. Sitagliptin has been used in People's Republic of China, and sitagliptin and metformin combination therapy may not alter their individual pharmacokinetics. To date, several clinical trials undertaken to investigate the efficacy of sitagliptin and metformin combination therapy have revealed that it effectively controlled glycated hemoglobin, fasting plasma glucose, and postprandial plasma glucose levels to a greater extent than sitagliptin or metformin alone. In addition, the combined therapy was well tolerated and induced few side effects, which were largely mild. Furthermore, the combined therapy was easy to administer, and the patients receiving this therapy showed good compliance. Therefore, for Chinese patients with type $2 \mathrm{DM}$, sitagliptin and metformin combination therapy is preferred.
\end{abstract}

Keywords: type 2 diabetes mellitus, sitagliptin, metformin

\section{Introduction}

The prevalence of diabetes mellitus (DM) continues to increase worldwide. ${ }^{1}$ In People's Republic of China, it has increased from $2.5 \%$ in $1994^{2}$ to $9.7 \%$ in $2010^{3}$ and $11.6 \%$ in $2013 .{ }^{4}$ At present, there are more than 100 million patients with DM in People's Republic of China. ${ }^{5}$ According to the Guideline for the Prevention and Treatment of Type 2 Diabetes Mellitus in China (2013), type 2 DM accounts for more than 90\% of the DM cases, while type $1 \mathrm{DM}$ accounts for approximately $5 \% .^{6}$ In addition, the proportion of patients diagnosed with prediabetes is as high as $15.5 \%,{ }^{3}$ and many patients continue to be undiagnosed. Available epidemiological studies indicate that the proportion of Chinese DM patients achieving glucose control is less than $30 \%,{ }^{3}$ suggesting that there is a lack of effective glucose-lowering therapies. Thus, it is imperative to develop an effective glucose-lowering protocol for Chinese patients.

The characteristics of DM in Asians are different from those in other areas of the world. ${ }^{7-9}$ For example, Chinese DM patients experience $\beta$ cell dysfunction ${ }^{10}$ and are more susceptible to increased postprandial plasma glucose ${ }^{11}$ in the early stages of DM. In addition, as compared to people in Western countries, the body weight of Asians is lower, and Chinese patients also exercise less. Furthermore, carbohydrates are a major component of each meal in People's Republic of China. These differences may affect the efficacy of glucose-lowering therapy. 
To date, the pathogenesis of DM has not been completely elucidated. In addition to the defective secretion of insulin by $\beta$ cells, insulin resistance, increased hepatic glucose output, and reduced glucose use in peripheral tissues, other factors, such as increased secretion of glucagon by $\alpha$ cells $^{12,13}$ and insufficiency and/or compromised function of the incretin hormone, especially including glucagon-like peptide-1 (GLP-1), ${ }^{14}$ are also involved in the pathogenesis of DM.

In 1964, Elrick et al ${ }^{15}$ found that oral glucose is more potent to promote the secretion of insulin as compared with intravenous glucose; this effect is known as the incretin effect. Incretins include GLP-1 and glucose-dependent insulin-releasing peptide; GLP-1 is more important for DM. GLP-1 has multiple activities in different organs/systems. It not only may promote the synthesis and secretion of insulin by $\beta$ cells but also stimulates $\beta$ cell proliferation and inhibits its apoptosis. ${ }^{16-19}$ In $\alpha$ cells, GLP-1 inhibits the release of glucagon. ${ }^{20}$ Furthermore, in the gastrointestinal tract, GLP-1 may delay gastric emptying, inhibit gastrointestinal peristalsis, and suppress appetite. ${ }^{21}$ These features of GLP-1 may influence the characteristics of Chinese DM patients.

Several minutes after food intake, GLP-1 is detected in the plasma. However, natural GLP-1 has a half-life of 1-2 minutes, and is rapidly degraded by dipeptidyl peptidase-4 (DPP-4). At present, there are two methods to increase plasma GLP-1: (i) direct injection of GLP-1 analogs (eg, liraglutide and exenatide) and (ii) oral intake of a DPP-4 inhibitor, including sitagliptin, vildagliptin, saxagliptin, and linagliptin. As a kind of DPP-4 inhibitor, sitagliptin has a longer half-life than other DPP-4 inhibitors.

\section{Pharmacokinetics of sitagliptin and metformin}

Sitagliptin, the first DPP-4 inhibitor used for DM, was approved by the US Food and Drug Administration in 2006 for the treatment of DM and by the State Food and Drug Administration of China in 2010. A single dose of oral sitagliptin may inhibit DPP-4 activity for 24 hours, increasing GLP-1 levels by two- to threefold, which results in increased insulin release, glucagon reduction, and decreased glucose levels. In addition, in a manner similar to that of glucosidase inhibitors, sitagliptin can delay intestinal absorption of carbohydrates, which helps to control postprandial plasma glucose and prevents hypoglycemia before the next meal. Moreover, several trials have confirmed that sitagliptin is protective for the heart ${ }^{22-24}$ and nervous system. ${ }^{25}$

The pharmacokinetics of sitagliptin is comparable between healthy subjects and type $2 \mathrm{DM}$ patients. The intraindividual and interindividual coefficients of variation in plasma sitagliptin concentration over time are small (5.8\% and $15.1 \%$, respectively). In healthy subjects, oral intake of $100 \mathrm{mg}$ of sitagliptin results in its rapid absorption; the plasma concentration $\left(T_{\max }\right)$ of sitagliptin peaked within 1-4 hours, and its half-life was 12.4 hours. The absolute bioavailability of sitagliptin is approximately $87 \%$. Because a high-fat diet does not influence the pharmacokinetics of sitagliptin, it can be taken with or without food. Sitagliptin is primarily cleared by the kidney $(\sim 87 \%)$ via active secretion in the renal tubules and excreted through the urine in its original form, ${ }^{26}$ the renal clearance of sitagliptin is approximately $350 \mathrm{~mL} / \mathrm{min}$. The remaining sitagliptin is excreted in the stool (13\%) within 1 week following oral intake. ${ }^{26}$

The mechanism by which metformin lowers plasma glucose levels includes the promotion of glucose use by peripheral cells (eg, muscle cells), inhibition of hepatic gluconeogenesis, and suppression of hepatic glucose output. Metformin is primarily absorbed in the small intestine. Under fasting conditions, the absolute bioavailability of metformin is $50 \%-60 \%$ following oral intake of $0.5 \mathrm{~g}$, and concomitant food intake may reduce the rate and extent of its absorption. The peak $\left(T_{\max }\right)$ of metformin is achieved after 2 hours, and the mean half-life is approximately 4 hours. Metformin may not bind plasma proteins. Stable $\left(T_{\max }\right)$ may be achieved within 24-48 hours after oral intake of metformin at routine doses and with a traditional protocol. Metformin is primarily excreted through the kidney $(\sim 90 \%)$ within 24 hours after oral intake. ${ }^{27}$

The feasibility of the combined use of a DPP-4 inhibitor with metformin has been described. ${ }^{28}$ The pharmacokinetic interaction between sitagliptin and metformin and tolerance of the combined therapy were investigated when stable plasma sitagliptin and metformin concentrations were achieved 7 days after intake. ${ }^{29}$ Subjects had good tolerance to the combined therapy, suggesting the absence of pharmacokinetic interaction (steady-state pharmacokinetics). Similarly, in type 2 DM patients, sitagliptin and metformin combination therapy had no influence on the pharmacokinetics of sitagliptin. ${ }^{26}$

\section{The efficacy of sitagliptin-metformin combination therapy in Chinese patients}

In People's Republic of China, several studies have been conducted to investigate the efficacy of sitagliptin and metformin combined therapy in type $2 \mathrm{DM}$ patients (Table 1). In 30 type 2 DM patients with poor glucose control following treatment 


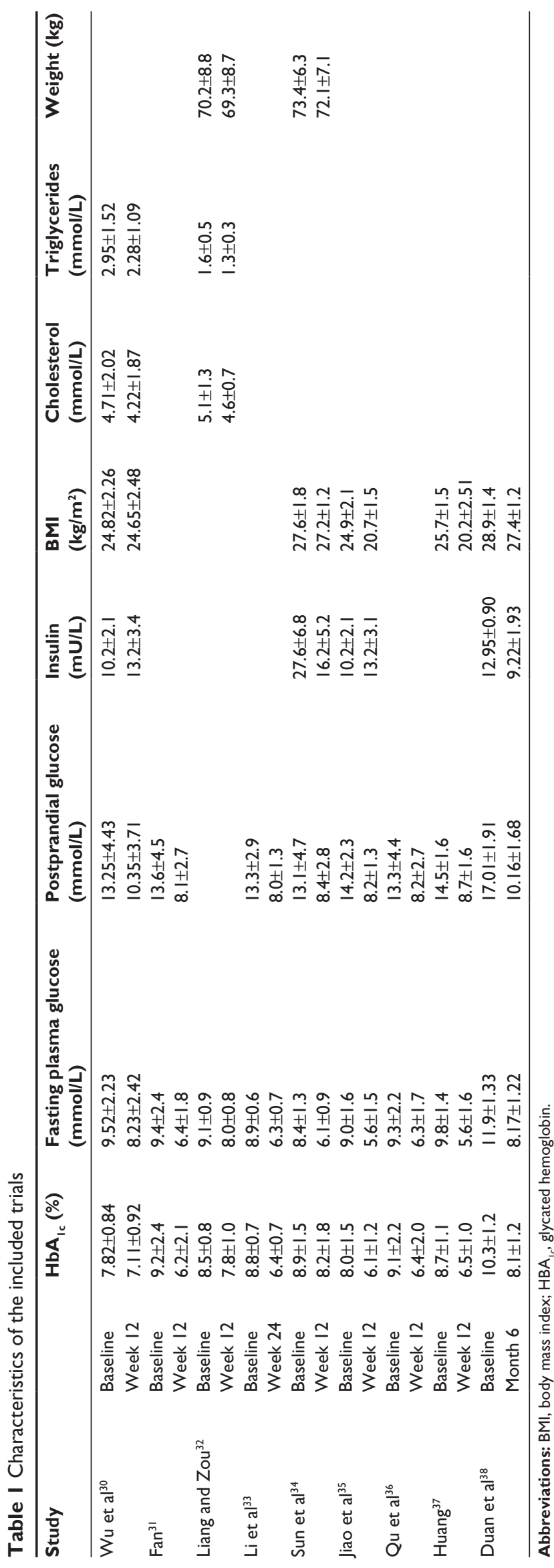

with metformin alone, combined therapy of sitagliptin and metformin for 12 weeks significantly reduced glycated hemoglobin, fasting plasma glucose, and postprandial glucose; however, the body mass index (BMI) of the patients remained unchanged ${ }^{30}$ Two patients complained of six hypoglycemic events; other adverse events included nausea $(n=3)$, vomiting $(\mathrm{n}=1)$, abdominal pain $(\mathrm{n}=1)$, fatigue $(\mathrm{n}=2)$, and dizziness $(\mathrm{n}=1)$. The side effects were mild and resolved spontaneously, and none of the patients withdrew from the study. ${ }^{30}$ In another study, 76 patients with type 2 DM were randomly divided into the following two treatment groups: sitagliptin plus metformin and pioglitazone plus metformin. ${ }^{28}$ After 12 weeks, glycated hemoglobin, fasting plasma glucose, and postprandial plasma glucose levels were significantly reduced in both groups; however, a greater reduction in glycated hemoglobin, fasting plasma glucose, and postprandial plasma glucose was observed in the sitagliptin group. ${ }^{31}$ Three patients reported a slight increase in alanine aminotransferase, four had diarrhea, and three developed hypoglycemia. ${ }^{31}$ In another study ${ }^{32}$ of 80 type 2 DM patients with poor plasma glucose control following metformin therapy alone, treatment with metformin plus sitagliptin significantly reduced glycated hemoglobin, fasting plasma glucose, plasma lipid, and body weight. Furthermore, in a study ${ }^{33}$ that included 116 patients with overweight and type $2 \mathrm{DM}$, who had poor glucose control after metformin monotherapy, combination therapy with metformin and sitagliptin or glimepiride for 24 weeks dramatically reduced glycated hemoglobin, fasting plasma glucose, and postprandial plasma glucose levels. Moreover, body weight was reduced in the sitagliptin group but was increased in the glimepiride group, and the incidence of hypoglycemia was lower in the sitagliptin group as compared with the glimepiride group. With the exception of hypoglycemia, no other side effects were reported. In 66 type $2 \mathrm{DM}$ patients with poor glucose control following metformin monotherapy, additional sitagliptin therapy for 12 weeks decreased body weight, BMI, and glycated hemoglobin levels although not to significant levels $(P>0.05) .{ }^{34}$ In addition, fasting plasma glucose and postprandial plasma glucose were markedly reduced, and four patients reported hypoglycemia after exercise, which was resolved after food intake. ${ }^{34}$ In another study that included 60 patients with newly onset type 2 DM who were randomly assigned to receive either metformin alone or metformin plus sitagliptin, combined therapy for 12 weeks significantly reduced fasting plasma glucose, postprandial plasma glucose, glycated hemoglobin, and BMI compared with metformin monotherapy. ${ }^{35}$ Two patients in the combined therapy group developed diarrhea, and headache $(n=1)$ and nausea $(n=2)$ 
were observed in the metformin monotherapy group. In 70 type $2 \mathrm{DM}$ patients randomly assigned to receive either metformin plus sitagliptin or metformin plus pioglitazone for 12 weeks, fasting and postprandial plasma glucose and glycated hemoglobin were significantly reduced. ${ }^{36}$ The reduction in postprandial plasma glucose was more evident in the sitagliptin group than in the pioglitazone group. In the sitagliptin group, two patients developed a slight increase in alanine aminotransferase, three patients had diarrhea, and two developed hypoglycemia. ${ }^{36}$ In the pioglitazone group, three patients developed a slight increase in alanine aminotransferase, two had diarrhea, and three developed hypoglycemia. In a study of 80 patients with newly onset type $2 \mathrm{DM}$, patients were randomly assigned to two treatment groups: metformin monotherapy and metformin plus sitagliptin combination therapy. ${ }^{37}$ After 12 weeks, those receiving the combined therapy had marked reductions in fasting and postprandial plasma glucose, glycated hemoglobin, and BMI compared with patients receiving metformin monotherapy. In the combined therapy group, three patients had diarrhea, two patients developed constipation, and nausea and vomiting were found in two patients. In the monotherapy group, nausea and vomiting were observed in five patients. ${ }^{37}$ In a study of 82 patients with new-onset type 2 DM randomly assigned to receive either metformin monotherapy or metformin plus sitagliptin combination therapy, BMI, glycated hemoglobin, and fasting and postprandial plasma glucose levels in patients receiving the combination therapy were significantly lower than those in the monotherapy group after 6 months. ${ }^{38}$

Taken together, combined therapy with sitagliptin and metformin effectively controls plasma glucose in type 2 DM patients as characterized by significant reductions in fasting and postprandial plasma glucose levels. With the exception of one study, ${ }^{34}$ glycated hemoglobin was also dramatically reduced followed the combined therapy. Thus, the glucoselowering effect of the combined therapy was superior to that of monotherapy with metformin or sitagliptin. Of note, changes in body weight following therapy varied among the studies with some studies suggesting that sitagliptin may promote metformin-induced weight loss, while other studies observed no effect on body weight. These varying effects on body weight are consistent with those reported in other countries. ${ }^{39-43}$ However, body weight gain was observed in lean subjects in a Japanese study. ${ }^{44}$ Following the combined therapy, common side effects included gastrointestinal reactions, hypoglycemia, and a slight increase in transaminase levels, which were usually mild and well tolerated by the patients. Of note, no study reported pancreatitis in patients receiving the combined therapy. Considering the relationship between sitagliptin and pancreatitis, ${ }^{45-48}$ although there is still controversy on this issue, ${ }^{48-51}$ it is necessary to investigate pancreatitis in type 2 DM patients treated with sitagliptin in further studies.

This review had some limitations. The ages of patients participating in these trials differed, and the course of the disease was also different. The sample sizes of the trials included in the review were different, and the objects and randomization of the trials were different. For example, the aims of the trials were to compare the efficacy of sitagliptin and metformin, sitagliptin and pioglitazone, or sitagliptin and glimepiride. Besides, combination of two drugs was taken in some trials in contrast to single-drug therapy. Moreover, there were differences in study design and methodology, which may influence clinical heterogeneity and cause bias in results affecting the conclusions.

\section{Patient satisfaction/acceptability, adherence, and uptake}

In addition to diet control and exercise, type $2 \mathrm{DM}$ is also managed with oral glucose-lowering drugs, subcutaneous injection of insulin, and subcutaneous injection of GLP-1 analogs. Oral glucose-lowering drugs, including biguanides, sulfonylureas, glycosidase inhibitors, thiazolidinediones, and non-sulfonylureas, promote insulin secretion and/or inhibit DPP-4. Metformin is the most common biguanide used in clinical practice. Although phenformin is still applied in the therapy of DM in several developing areas, physicians should emphasize that it may cause lactic acidosis. ${ }^{52}$ Sulfonylureas used for the therapy of DM include glimepiride, gliclazide, gliquidone, and glipizide. In addition, glibenclamide, a sulfonylurea with a long half-life, ${ }^{53}$ may be used, although it is known to cause severe hypoglycemia. Glucosidase inhibitors include acarbose, voglibose, and miglitol, and thiazolidinediones include rosiglitazone and pioglitazone. Non-sulfonylureas that promote insulin secretion include repaglinide, nateglinide, and mitiglinide. Furthermore, DPP-4 inhibitors include sitagliptin, saxagliptin, vildagliptin, linagliptin, and alogliptin. In addition to human insulin, there are some insulin analogs used for DM, including glargine, ${ }^{54}$ detemir, ${ }^{55}$ aspart, ${ }^{56}$ lispro, ${ }^{57}$ and degludec. ${ }^{58}$ Generally, premixed insulin is administered subcutaneously twice daily before breakfast and supper or thrice daily before each meal. ${ }^{59}$ Usually, treatment of premixed insulin includes one injection of intermediate-to-long-acting insulin and three injections of short-acting insulin before meals. GLP-1 analogs include exenatide ${ }^{60}$ and liraglutide, ${ }^{61}$ and are usually administered subcutaneously once or twice daily. 
In addition, insulin, oral glucose-lowering drugs, and GLP-1 analogs can be combined to identify an optimal treatment protocol for each patient.

Sitagliptin is currently used in the therapy of type 2 DM in People's Republic of China. Although it is more widely used in clinical practice compared with other DPP-4 inhibitors, its use is not widespread. In some large cities, sitagliptin is frequently prescribed in comprehensive hospitals. In addition, oral administration of sitagliptin is more convenient compared with subcutaneous injection of GLP-1 analogs. Thus, sitagliptin is the preferred incretin therapeutic. However, in small cities or small hospital or clinics, sitagliptin is less frequently used, and may not be available in some pharmacies. Moreover, many patients have little knowledge of sitagliptin.

For type 2 DM patients, sitagliptin $(100 \mathrm{mg})$ is administered orally once daily. Patients are usually satisfied with its therapeutic efficacy, and few side effects are observed; thus, patients often have good compliance and tolerance to sitagliptin. For patients with good glucose control, $50 \mathrm{mg}$ of oral sitagliptin may be administered once daily. In addition, for patients with kidney dysfunction, creatinine clearance should be calculated, and the dose be adjusted according to creatinine clearance. There are no studies at present regarding sitagliptin use by pregnant or breast-feeding patients and those younger than 18 years.

Metformin is a glucose-lowering drug that has been used for more than 50 years and is widely used in the therapy of type 2 DM patients in People's Republic of China. It is the first treatment of choice for type $2 \mathrm{DM}$ when contradictions are not indicated. Metformin is often available in general hospitals and pharmacies and is also inexpensive. The total daily dose of metformin is $1,500-2,500 \mathrm{mg}$, which is administered several times throughout the day. Generally, $500 \mathrm{mg}$ metformin is administered thrice daily. In addition, the dose of metformin should be adjusted according to plasma glucose levels and body weight. The side effects associated with metformin treatment are often present in the gastrointestinal system, and some patients can tolerate them well enough to continue metformin therapy. However, a fraction of patients cannot tolerate these side effects, and other therapeutic protocols are used in these cases.

$\mathrm{DM}$ is a great threat to human health and represents a huge burden to both developed and developing countries. ${ }^{62}$ To develop individualized therapeutic protocols according to the characteristics of DM in a specific country will be of great benefit in the treatment of DM. In cases in which metformin monotherapy fails to adequately control plasma glucose, the combined use of sitagliptin is recommended as it may exert synergistic effects to improve insulin sensitivity and $\beta$ cell function and reduce glucose without affecting individual pharmacokinetics. The multiple effects of sitagliptin are applicable for the treatment of DM in Chinese patients, especially given that combination therapy was associated with mild side effects, which were well tolerated by the patients. In addition, this combination therapy is convenient to administer and cost-effective. Moreover, sitagliptin can be used in combination with other drugs, such as sulfonylurea, ${ }^{63}$ non-sulfonylureas that promote insulin secretion, glucosidase inhibitors, ${ }^{64}$ and insulin, ${ }^{65}$ as well as metformin.

\section{Conclusion}

Sitagliptin has been used for the management of type 2 DM in People's Republic of China for more than 3 years. Clinical studies suggest that sitagliptin in combination with metformin is superior to metformin monotherapy in controlling plasma glucose levels. In addition, this combination therapy is safe, convenient to administer, cost-effective, and well tolerated; therefore, patients are often compliant. Thus, it is a preferred strategy for the treatment of type $2 \mathrm{DM}$, and it is recommended to add sitagliptin after metformin. A correlation between sitagliptin and reduced body weight and the incidence of pancreatitis has been noted in a few studies, which should be confirmed in additional clinical studies. Moreover, more randomized, controlled studies with larger sample sizes are required to confirm the efficacy of this treatment strategy for type $2 \mathrm{DM}$.

\section{Disclosure}

The authors report no conflicts of interest in this work.

\section{References}

1. Unwin N, Guariguata L, Whiting D, Weil C. Complementary approaches to estimation of the global burden of diabetes. Lancet. 2012;379(9825): 1487-1488

2. Pan XR, Yang WY, Li GW, Liu J. Prevalence of diabetes and its risk factors in China, 1994. National Diabetes Prevention and Control Cooperative Group. Diabetes Care. 1997;20(11):1664-1669.

3. Yang W, Lu J, Weng J, et al; China National Diabetes and Metabolic Disorders Study Group. Prevalence of diabetes among men and women in China. N Engl J Med. 2010;362(12):1090-1101.

4. Xu Y, Wang L, He J, et al; 2010 China noncommunicable disease surveillance Group. Prevalence and control of diabetes in Chinese adults. JAMA. 2013;310(9):948-959.

5. Ma RC, Lin X, Jia W. Causes of type 2 diabetes in China. Lancet Diabetes Endocrinol. 2014;2(12):70145-70147.

6. Chinese Diabetes Society. Guideline of prevention and treatment for type 2 diabetes patients in China. China J Diabetes. 2014;22(8):2-43.

7. Lin JD, Chen YL, Hsu CH, et al. Beta-cell function and insulin sensitivity at various degrees of glucose tolerance in Chinese subjects. Diabetes Res Clin Pract. 2013;100(3):391-397.

8. Hong J, Gu WQ, Zhang YF, et al. The interplay of insulin resistance and beta-cell dysfunction involves the development of type 2 diabetes in Chinese obeses. Endocrine. 2007;31(2):93-99. 
9. Tuomi T, Santoro N, Caprio S, Cai M, Weng J, Groop L. The many faces of diabetes: a disease with increasing heterogeneity. Lancet. 2014; 383(9922):1084-1094.

10. Yang W, Weng J. Early therapy for type 2 diabetes in China. Lancet Diabetes Endocrinol. 2014;2(12):70136-70146.

11. Bi Y, Zeng L, Zhu D, et al. Association of beta-cell function and insulin sensitivity with fasting and 2-h plasma glucose in a large Chinese population. Diabetes Obes Metab. 2012;14(2):174-180.

12. Cryer PE. Minireview: glucagon in the pathogenesis of hypoglycemia and hyperglycemia in diabetes. Endocrinology. 2012;153(3): 1039-1048.

13. Farhy LS, McCall AL. Optimizing reduction in basal hyperglucagonaemia to repair defective glucagon counterregulation in insulin deficiency. Diabetes Obes Metab. 2011;Suppl 1:133-143.

14. Gerich J. Pathogenesis and management of postprandial hyperglycemia: role of incretin-based therapies. Int J Gen Med. 2013;6:877-895.

15. Elrick H, Stimmler L, Hlad CJ Jr, Arai Y. Plasma insulin response to oral and intravenous glucose administration. J Clin Endocrinol Metab. 1964;24:1076-1082.

16. Lee YS, Jun HS. Anti-diabetic actions of glucagon-like peptide-1 on pancreatic beta-cells. Metabolism. 2014;63(1):9-19.

17. Poucher SM, Cheetham S, Francis J, Zinker B, Kirby M, Vickers SP. Effects of saxagliptin and sitagliptin on glycaemic control and pancreatic $\beta$-cell mass in a streptozotocin-induced mouse model of type 2 diabetes. Diabetes Obes Metab. 2012;14(10):918-926.

18. Kim YS, Oh SH, Park KS, et al. Improved outcome of islet transplantation in partially pancreatectomized diabetic mice by inhibition of dipeptidyl peptidase-4 with sitagliptin. Pancreas. 2011;40(6):855-860.

19. Takeda Y, Fujita Y, Honjo J, et al. Reduction of both beta cell death and alpha cell proliferation by dipeptidyl peptidase- 4 inhibition in a streptozotocin-induced model of diabetes in mice. Diabetologia. 2012; 55(2):404-412.

20. Drucker DJ. Incretin action in the pancreas: potential promise, possible perils, and pathological pitfalls. Diabetes. 2013;62(10):3316-3323.

21. Unger J. Rationale use of GLP-1 receptor agonists in patients with type 1 diabetes. Curr Diab Rep. 2013;13(5):663-668.

22. Tremblay AJ, Lamarche B, Deacon CF, Weisnagel SJ, Couture P. Effects of sitagliptin therapy on markers of low-grade inflammation and cell adhesion molecules in patients with type 2 diabetes. Metabolism. 2014;63(9):1141-1148.

23. McCormick LM, Kydd AC, Read PA, et al. Chronic dipeptidyl peptidase-4 inhibition with sitagliptin is associated with sustained protection against ischemic left ventricular dysfunction in a pilot study of patients with type 2 diabetes mellitus and coronary artery disease. Circ Cardiovasc Imaging. 2014;7(2):274-281.

24. Zeng Y, Li C, Guan M, et al. The DPP-4 inhibitor sitagliptin attenuates the progress of atherosclerosis in apolipoprotein-E-knockout mice via AMPK- and MAPK-dependent mechanisms. Cardiovasc Diabetol. 2014;13:32.

25. Sharma AK, Sharma A, Kumari R, et al. Sitagliptin, sitagliptin and metformin, or sitagliptin and amitriptyline attenuate streptozotocinnicotinamide induced diabetic neuropathy in rats. $J$ Biomed Res. 2012;26(3):200-210.

26. Januvia ${ }^{\circledR}$ (sitagliptin) tablets [prescribing information]. Whitehouse Station, NJ: Merck \& Co., Inc. Available from: http://www.accessdata. fda.gov/drugsatfda_docs/label/2012/021995s023lbl.pdf. Accessed November 1, 2014.

27. GLUCOPHAGE ${ }^{\circledR}$ (metformin hydrochloride) tablets, GLUCOPHAGE ${ }^{\circledR}$ XR (metformin hydrochloride) extended-release tablets [prescribing information]. Princeton, NJ: Bristol-Myers Squibb Company. Available from: http://www.accessdata.fda.gov/drugsatfda_docs/label/2008/020 357s031,021202s0161bl.pdf. Accessed November 1, 2014.

28. Herman GA, Bergman A, Yi B, Kipnes M; Sitagliptin Study 012 Group. Tolerability and pharmacokinetics of metformin and the dipeptidyl peptidase-4 inhibitor sitagliptin when co-administered in patients with type 2 diabetes. Curr Med Res Opin. 2006;22(10):1939-1947.
29. Fass AD, Gershman JA. Efficacy and safety of dipeptidyl peptidase-4 inhibitors in combination with metformin. Adv Ther. 2013;30(4): 337-353.

30. Wu DY, MA WQ, Wang LG, et al. Efficacy of dipeptidyl peptidase-4 inhibitor sitagliptin on patients with type 2 diabetes inadequately controlled with metformin alone. Clinical Focus. 2011;26(20):1762-1764. Chinese.

31. Fan XX. Clinical efficacy of metformin combined with sitagliptin or pioglitazone in treatment of type 2 diabetes mellitus. Clin Med China. 2013;29(12):1295-1298. Chinese.

32. Liang JJ, Zou DJ. Cost-effectiveness analysis of sitagliptin and pioglitazone in poorly controlled type 2 diabetes mellitus patients with oral metformin. Shanghai Med J. 2013;36(5):414-417. Chinese.

33. Li WH, Lin M, Zhang XJ. Comparison on adding sitagliptin or glimepiride in poorly controlled overweight type 2 diabetes with oral metformin. Chin Hosp Pharm J. 2012;32(10):792-794. Chinese.

34. Sun J, Li SS, Jiang S, Quan L. Efficacy of sitagliptin controlled with metformin on patients with type 2 diabetes mellitus. Chinese J Prac Med. 2013;40(19):105-106. Chinese.

35. Jiao XM, Xu XP, Zhao J, Lv XF. Clinical observation on treatment of newly diagnosed type 2 diabetes with sitagliptin and metformin. Clin Focus. 2013;28(1):44-48. Chinese.

36. Qu Y, Zhang CL, Hu WJ, Liu MY, He R. Clinical observation on curative effect of sitagliptin, rosiglitazone combined with Metformin respectively in treatment of elderly type 2 diabetes. Prog Mod Biomed. 2013;13(21):4077-4080. Chinese.

37. Huang BX. Clinical observation on treatment of newly diagnosed diabetes with sitagliptin and metformin. Nat Med Front China. 2011; 6(6):58-59. Chinese.

38. Duan LJ, Zhang Y, Jiang X. Efficacy studies of sitagliptin combined with metformin in newly diagnosed type 2 diabetes patients. Clin Med China. 2013;29(4):379-382. Chinese.

39. Traina S, Guthrie R, Slee A. The impact of weight loss on weight-related quality of life and health satisfaction: results from a trial comparing canagliflozin with sitagliptin in triple therapy among people with type 2 diabetes. Postgrad Med. 2014;126(3):7-15.

40. Montilla S, Marchesini G, Sammarco A, et al; AIFA Anti-diabetics Monitoring Group. Drug utilization, safety, and effectiveness of exenatide, sitagliptin, and vildagliptin for type 2 diabetes in the real world: data from the Italian AIFA Anti-diabetics Monitoring Registry. Nutr Metab Cardiovasc Dis. 2014;24(12):1346-1353.

41. Wang T, Gou Z, Wang F, Ma M, Zhai SD. Comparison of GLP-1 an alogues versus sitagliptin in the management of type 2 diabetes: systematic review and meta-analysis of head-to-head studies. PLoS One. 2014;9(8):e103798.

42. Ahrén B, Johnson SL, Stewart M, et al; HARMONY 3 Study Group. HARMONY 3: 104-week randomized, double-blind, placebo- and active-controlled trial assessing the efficacy and safety of albiglutide compared with placebo, sitagliptin, and glimepiride in patients with type 2 diabetes taking metformin. Diabetes Care. 2014;37(8): 2141-2148

43. Jung JA, Kaku K, Kim JH, et al. Additive postprandial glucose-lowering effects of mitiglinide and sitagliptin in patients with type 2 diabetes mellitus. Adv Ther. 2013;30(11):1018-1129.

44. Eiji K, Tadataka H. Effect of sitagliptin in type 1 or type 2 diabetic patients with absolute insulin deficiency: a 48 weeks observational study. Br J Med Med Res. 2013;3(4):1910-1917.

45. Scheen A. Gliptins (dipeptidyl peptidase-4 inhibitors) and risk of acute pancreatitis. Expert Opin Drug Saf. 2013;12(4):545-557.

46. Iyer SN, Drake AJ 3rd, West RL, Mendez CE, Tanenberg RJ. Case report of acute necrotizing pancreatitis associated with combination treatment of sitagliptin and exenatide. Endocr Pract. 2012;18(1):e10-e13.

47. Mondragon A, Davidsson D, Kyriakoudi S, et al. Divergent effects of liraglutide, exendin-4, and sitagliptin on beta-cell mass and indicators of pancreatitis in a mouse model of hyperglycaemia. PLoS One. 2014;9(8):e104873. 
48. Faillie JL, Babai S, Crépin S, et al; French Pharmacovigilance Centers Network. Pancreatitis associated with the use of GLP-1 analogs and DPP-4 inhibitors: a case/non-case study from the French Pharmacovigilance Database. Acta Diabetol. 2014;51(3):491-497.

49. Li L, Shen J, Bala MM, et al. Incretin treatment and risk of pancreatitis in patients with type 2 diabetes mellitus: systematic review and meta-analysis of randomised and non-randomised studies. BMJ. 2014;348:g2366.

50. Plosker GL. Sitagliptin: a review of its use in patients with type 2 diabetes mellitus. Drugs. 2014;74(2):223-242.

51. Monami M, Dicembrini I, Mannucci E. Dipeptidyl peptidase-4 inhibitors and pancreatitis risk: a meta-analysis of randomized clinical trials. Diabetes Obes Metab. 2014;16(1):48-56.

52. Fimognari FL, Pastorelli R, Incalzi RA. Phenformin-induced lactic acidosis in an older diabetic patient: a recurrent drama (phenformin and lactic acidosis). Diabetes Care. 2006;29(4):950-951.

53. Veitch PC, Clifton-Bligh RJ. Long-acting sulfonylureas - long-acting hypoglycaemia. Med J Aust. 2004;180(2):84-85.

54. Rendell M, Akturk HK, Tella SH. Glargine safety, diabetes and cancer. Expert Opin Drug Saf. 2013;12(2):247-263.

55. Keating GM. Insulin detemir: a review of its use in the management of diabetes mellitus. Drugs. 2012;72(17):2255-2287.

56. Heller S, McCance DR, Moghissi E, Nazeri A, Kordonouri O. Diversity in diabetes: the role of insulin aspart. Diabetes Metab Res Rev. 2012; 28(1):50-61

57. Vincent M, Nobécourt E. Treatment of diabetic ketoacidosis with subcutaneous insulin lispro: a review of the current evidence from clinical studies. Diabetes Metab. 2013;39(4):299-305.

58. Philis-Tsimikas A, BrodM, Niemeyer M, Ocampo Francisco AM, Rothman J. Insulin degludec once-daily in type 2 diabetes: simple or step-wise titration (BEGIN: once simple use). Adv Ther. 2013;30(6):607-622.
59. Yang W, Ji Q, Zhu D, et al. Biphasic insulin aspart 30 three times daily is more effective than a twice-daily regimen, without increasing hypoglycemia, in Chinese subjects with type 2 diabetes inadequately controlled on oral antidiabetes drugs. Diabetes Care. 2008;31(5):852-856.

60. Parkes DG, Mace KF, Trautmann ME. Discovery and development of exenatide: the first antidiabetic agent to leverage the multiple benefits of the incretin hormone, GLP-1. Expert Opin Drug Discov. 2013;8(2): 219-244.

61. Nauck M, Frid A, Hermansen K, et al. Long-term efficacy and safety comparison of liraglutide, glimepiride and placebo, all in combination with metformin in type 2 diabetes: 2-year results from the LEAD-2 study. Diabetes Obes Metab. 2013;15(3):204-212.

62. Chan JC, Zhang Y, Ning G. Diabetes in China: a societal solution for a personal challenge. Lancet Diabetes Endocrinol. 2014;2(14):969-979.

63. Arai K, Maeda H, Sirabe S, et al. Glimepiride strongly enhances the glucose-lowering effect in triple oral antidiabetes therapy with sitagliptin and metformin for Japanese patients with type 2 diabetes mellitus. Diabetes Technol Ther. 2013;15(4):335-341.

64. Osonoi T, Saito M, Tamasawa A, Ishida H, Osonoi Y. Effects of sitagliptin or mitiglinide as an add-on to acarbose on daily blood glucose fluctuations measured by $72 \mathrm{~h}$ subcutaneous continuous glucose monitoring in Japanese patients with type 2 diabetes: a prospective randomized study. Expert Opin Pharmacother. 2014;15(10):1325-1335.

65. Barnett AH. Complementing insulin therapy to achieve glycemic control. Adv Ther. 2013;30(6):556-557.
Patient Preference and Adherence

\section{Publish your work in this journal}

Patient Preference and Adherence is an international, peer-reviewed, open access journal that focuses on the growing importance of patient preference and adherence throughout the therapeutic continuum. Patient satisfaction, acceptability, quality of life, compliance, persistence and their role in developing new therapeutic modalities and compounds to optimize

\section{Dovepress}

clinical outcomes for existing disease states are major areas of interest for the journal. This journal has been accepted for indexing on PubMed Central. The manuscript management system is completely online and includes a very quick and fair peer-review system, which is all easy to use. Visit http://www. dovepress.com/testimonials.php to read real quotes from published authors. 